\title{
On the derived category of $\bar{M}_{0, n}$
}

\author{
Yu. Manin, M. Smirnov \\ Max-Planck-Institut für Mathematik, Bonn, Germany
}

ABSTRACT. Using Keel's presentation and Orlov's theorem, we give an inductive description of the derived category of moduli spaces of $n$-pointed stable curves of genus zero and some full exceptional collections in it. The detailed calculations are given for $\bar{M}_{0,6}$.

\section{Introduction}

0.1. Summary. Let $f: X \rightarrow Y$ be a monoidal transformation of smooth projective schemes with smooth center $Z \subset Y$. Then $D^{b}(C o h X)$ in a definite sense can be described in terms of $D^{b}(\operatorname{Coh} Y)$ and $D^{b}($ Coh $Z)$ : cf. [Or1].

We apply this strategy to the calculation of the derived categories of moduli spaces $\bar{M}_{0, n}$. Since there are several methods of representing $\bar{M}_{0, n}$ as the result of a sequence of blow-ups with controlled bases and centers (see e.g. [Ke] and [Ka]), one may later ask about the interaction of the results obtained etc.

In this note we focus on the representation discovered and used by Keel in [Ke]. Its remarkable feature is that $\bar{M}_{0, n+1}$ is obtained from $\bar{M}_{0, n} \times \bar{M}_{0,4}$ by iterated blow-ups of manifolds isomorphic to $\bar{M}_{0, p+1} \times \bar{M}_{0, q+1}, p+q=n$. This produces a straightforward inductive description if one complements the tools used in the computation a version of "Künneth formula" for the derived categories. The simplest useful form is furnished by Proposition 2.1.18 in [Bö] constructing exceptional collections on $V \times W$ from those on $V$ and $W$. A much more sophisticated version is contained in [BoLaLu]; cf. also [Kel].

This approach might eventually furnish a concise and functorial description of quivers useful for computations of (and in) $D^{b}\left(\operatorname{Coh} \bar{M}_{0, n}\right)$, but we could not achieve this goal so far.

We imagine two directions of future work on this subject.

(A) We might expect that replacing motives of $\bar{M}_{0, S}$ by their derived (or enhanced derived) categories, we can get a better understanding of their quantum cohomology: cf. [MaS].

(B) The moduli spaces $\bar{M}_{0, S}$ are rigid as objects of commutative geometry. Ample experience, including Gauss' $q$-numbers and quantum groups, shows that noncommutative ("quantum") deformations of rigid objects are especially interesting. The beautiful recent paper [KeTe] shows how to represent $\bar{M}_{0, S}$ as the projective 
spectrum of a Koszul graded ring of sections of its log-canonical bundle. It would be interesting to study the deformations of the derived category of $\bar{M}_{0, S}$ in terms of the deformations of this coordinate ring, as well as in terms of Bondal's quivers [Bo].

See $[\mathrm{AuKaOr}]$ for a detailed discussion of the case of del Pezzo surfaces, containing in particular $\bar{M}_{0,5}$.

Noncommutative deformations of general $\bar{M}_{0, S}$, together with structural correspondences between them, that we envision, might become a sophisticated counterpart of the theory of quantum groups.

0.1. Plan of the paper. The first section summarizes the main theorem due to Orlov ([Or1]) allowing one to reconstruct the derived category of a blowup. We supplement it by some computations in the derived category, stressing, in particular, the codimension two blow-ups, that will appear in the applications to $\bar{M}_{0, S}$. The second section describes Keel's tower from [Ke]. In the third section the inductive construction based on the results of Orlov and Keel is elaborated. It is complemented by a new result (Proposition 3.5 and Corollary 3.6) allowing us in certain circumstances to get full exceptional collections consisting of locally free sheaves. Finally, sec. 4 gives an application of this construction to $\bar{M}_{0,6}$.

Acknowledgements. We are very grateful to D. Orlov for detailed and stimulating answers to the questions of Yu. M. Our thanks are due to Ch. Böhning for a discussion of "Kunneth formula" for derived categories.

\section{Orlov's theorem}

1.1. Exceptional collections. Let $K$ be a ground field; $D$ a $K$-linear triangulated category; $T$ its shift functor; $E, F \in O b D$. Following [Bo], we consider the graded complex of $K$-vector spaces with trivial differential

$$
\operatorname{Hom}_{D}^{\bullet}(E, F):=\bigoplus_{k \in \mathbf{Z}} \operatorname{Hom}_{D}^{k}(E, F)[-k], \quad \operatorname{Hom}_{D}^{k}(E, F):=\operatorname{Hom}_{D}\left(E, T^{k} F\right) .
$$

Remind the following definitions.

(i) An object $E$ is called exceptional one, if $\operatorname{Hom}_{D}^{\bullet}(E, E)$ consists of $K \cdot \operatorname{id}_{E}$ put in degree zero.

(ii) A family of exceptional objects $\left(E_{\alpha}\right)$ indexed by a totally ordered set of subscripts $\alpha$ is called an exceptional collection (or an exceptional sequence), if

$$
\operatorname{Hom}_{D}^{\bullet}\left(E_{\alpha}, E_{\beta}\right)=0 \quad \text { for } \quad \alpha>\beta
$$


It is called full, if it generates $D$.

(iii) An exceptional sequence $\left(E_{\alpha}\right)$ as above is called strong, if for any $\alpha<\beta$ the complex $\operatorname{Hom}_{D}^{\bullet}\left(E_{\alpha}, E_{\beta}\right)$ has at most one non-vanishing component which is then of degree zero.

1.2. Blow-ups. Let $X$ be a smooth projective variety over $K, Y \subset X$ its smooth closed subvariety, $j: Y \rightarrow X$ the respective embedding. We consider the diagram describing the monoidal transformation of $X$ with center $Y$ :

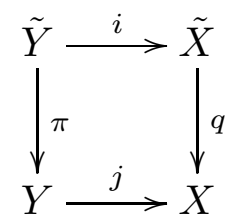

Let $\mathcal{N}$ be the sheaf of sections of the normal bundle to $Y$ in $X$. The exceptional divisor $\widetilde{Y}$ in $\widetilde{X}$ is canonically isomorphic to the relative projective spectrum of the symmetric algebra $S_{\mathcal{O}_{Y}}\left(\mathcal{N}^{t}\right)$ of the dual sheaf of sections of $N$. The rank $c \geq 2$ of $N$ equals to the codimension of $Y$; fibers of $\pi$ are $\mathbf{P}^{c-1}$. This projective bundle carries the standard invertible relative sheaves $\mathcal{O}_{\pi}(l)$.

From the general definitions, we obtain the canonical exact sequences

$$
0 \rightarrow \mathcal{N}^{t} \rightarrow j^{*}\left(\Omega_{X}^{1}\right) \rightarrow \Omega_{Y}^{1} \rightarrow 0
$$

and

$$
0 \rightarrow \pi^{*}\left(\Omega_{Y}^{1}\right) \rightarrow \Omega_{\widetilde{Y}}^{1} \rightarrow \Omega_{\widetilde{Y} / Y}^{1} \rightarrow 0
$$

The dual exact sequence to that in [Huy], p. 252, reads

$$
0 \rightarrow \Omega_{\widetilde{Y} / Y}^{1}(1) \rightarrow \pi^{*}\left(\mathcal{N}^{t}\right) \rightarrow \mathcal{O}_{\pi}(1) \rightarrow 0
$$

1.3. From exceptional collections on $X$ and $Y$ to those on $\tilde{Y}$ and $\widetilde{X}$. Generally, for a variety $Z$ we denote by $D(Z)$ the bounded derived category $D^{b}(\operatorname{Coh} Z)$.

Let $\left(E_{\alpha}\right), \alpha \in A$, be an exceptional collection in $D(X)$ and $\left(F_{\beta}\right), \beta \in B$, be an exceptional sequence in $D(Y)$. Consider the following sequence of objects in $D(\widetilde{X})$, that is indexed by the set of pairs $(\beta, l)$ for $\beta \in B,-c+1 \leq l \leq-1$ and pairs $(\alpha, l=0)$ for $\alpha \in A$ :

$$
R i_{*}\left(L \pi^{*}\left(F_{\beta}\right) \otimes \mathcal{O}_{\pi}(-c+1)\right), \ldots, R i_{*}\left(L \pi^{*}\left(F_{\beta}\right) \otimes \mathcal{O}_{\pi}(-1)\right), L q^{*}\left(E_{\alpha}\right) .
$$


It is ordered as suggested in (1.6): in each group with fixed $l$ according to the order of $A$ or $B$, and groups are ordered by increasing $l$.

1.4. Proposition. (i) The sequence (1.6) is exceptional.

(ii) If $\left(E_{\alpha}\right)$ is full in $D(X)$ and $\left(F_{\beta}\right)$ is full in $D(Y)$, then (1.6) is full in $D(\tilde{X})$.

This is the Corollary 4.4 in [Or1] (notice that our notation for the blow-up diagram (1.2) differs from Orlov's notation and is closer to the Huybrechts notation in [Huy], Ch. 11).

In the remaining part of this section we produce some formulas for morphism spaces between remaining pairs of objects in (1.6).

1.5. Auxiliary results. (a) Adjunction/duality formula. For a morphism $f: U \rightarrow V$ of smooth varieties, $\operatorname{define} \operatorname{dim} f:=\operatorname{dim} U-\operatorname{dim} V$ and put

$$
\omega_{f}:=\omega_{U} \otimes f^{*}\left(\omega_{V}^{-1}\right) .
$$

Then for $F \in D(U), E \in D(V)$ we have functorial isomorphisms ([Huy], p. 87)

$$
\operatorname{Hom}_{D(V)}\left(R f_{*}(F), E\right) \cong \operatorname{Hom}_{D(U)}\left(F, L f^{*}(E) \otimes \omega_{f}[\operatorname{dim} f]\right)
$$

(tensor products by an invertible or more general locally free sheaf here and below need not be derived).

(b) The sheaf $\omega_{\widetilde{Y}}$. We will prove that

$$
\omega_{\widetilde{Y}} \cong \pi^{*} \circ j^{*}\left(\omega_{X}\right) \otimes \mathcal{O}_{\pi}(-c) .
$$

In fact, from (1.4) and (1.3) we get

$$
\omega_{\widetilde{Y}} \cong \pi^{*}\left(j^{*}\left(\omega_{X}\right) \otimes \operatorname{det} \mathcal{N}\right) \otimes \omega_{\widetilde{Y} / Y}
$$

From (1.5) we obtain

$$
\pi^{*}(\operatorname{det} \mathcal{N}) \cong \omega_{\tilde{Y} / Y}^{-1} \otimes \mathcal{O}_{\pi}(-c) .
$$

Substituting (1.11) to (1.10), we get (1.9).

(c) The sheaf $\omega_{i}$. We wish to prove that

$$
\omega_{i} \cong \mathcal{O}_{\pi}(-1), \quad \omega_{i}[\operatorname{dim} i] \cong \mathcal{O}_{\pi}(-1)[-1] .
$$

In fact, in the notation of diagram (1.2), we have

$$
\omega_{\widetilde{X}} \cong q^{*}\left(\omega_{X}\right) \otimes \mathcal{O}_{\widetilde{X}}((c-1) \widetilde{Y}), \quad i^{*}\left(\mathcal{O}_{\widetilde{X}}(\widetilde{Y})\right) \cong \mathcal{O}_{\pi}(-1)
$$


where we write $\widetilde{Y}$ in place of the exceptional divisor $i(\widetilde{Y})$ : cf. [Huy], p. 252.

From (1.7) and (1.13) we find that

$$
\omega_{i}=\omega_{\widetilde{Y}} \otimes i^{*}\left(\omega_{\widetilde{X}}^{-1}\right) \cong \omega_{\widetilde{Y}} \otimes i^{*} \circ q^{*}\left(\omega_{X}^{-1}\right) \otimes \mathcal{O}_{\pi}(c-1) .
$$

Since $i^{*} \circ q^{*}=\pi^{*} \circ j^{*}$, substituting (1.9) into (1.14), we find (1.12).

(d) The sheaf $\omega_{\pi}$. From $(1.11)$ we can calculate $\omega_{\pi}:=\omega_{\tilde{Y}} \otimes \pi^{*}\left(\omega_{Y}^{-1}\right) \cong \omega_{\tilde{Y} / Y}$ :

$$
\omega_{\pi} \cong \operatorname{det} \pi^{*}(\mathcal{N})^{-1} \otimes \mathcal{O}_{\pi}(-c)
$$

and

$$
\omega_{\pi}[\operatorname{dim} \pi] \cong \pi^{*}(\operatorname{det} \mathcal{N})^{-1} \otimes \mathcal{O}_{\pi}(-c)[c-1] .
$$

(e) The sheaf $\omega_{j}$. We have

$$
\omega_{j} \cong \operatorname{det} \mathcal{N}, \quad \omega_{j}[\operatorname{dim} j] \cong \operatorname{det} \mathcal{N}[-c] .
$$

This follows directly from (1.3).

(f) The sheaf $\omega_{q}$. Finally, from (1.7) and (1.13) we get

$$
\omega_{q} \cong \mathcal{O}_{\widetilde{X}}((c-1) \widetilde{Y}) \cong \omega_{q}[\operatorname{dim} q]
$$

1.6. Calculations of Hom's. Below we will need some formulas for the morphism spaces between objects of the sequence (1.6) that do not follow directly from the exceptionality of this sequence, i. e. $\operatorname{Hom}_{D(\widetilde{X})}^{\bullet}(G, H)$ where $G, H$ are objects of (1.6), indexed respectively by $\left(\beta_{1}, l_{1}\right)$ and $\left(\beta_{2}, l_{2}\right)$ with $l_{1}<l_{2}$, or else by $(\beta, l), l<0$, and $(\alpha, 0)$. In fact, as Orlov has proved, for other pairs the respective Hom's come from $Y, X$ or vanish.

Moreover, in our applications to Keel's tower, all centers of consecutive blow-ups have codimension $c=2$. In this case the only relevant value of $l$ is $l=-1$ so that we need to consider only the second option.

Here we take two arbitrary objects $E \in D(X)$ and $F \in D(Y)$.

1.7. Proposition. There are functorial in E, F isomorphisms

$$
\begin{gathered}
\operatorname{Hom}_{D(\widetilde{X})}\left(R i_{*}\left(L \pi^{*}(F) \otimes \mathcal{O}_{\pi}(l)\right), L q^{*}(E)\right) \cong \\
\left.\operatorname{Hom}_{D(Y)}\left(F \otimes S_{\mathcal{O}_{Y}}^{-l-1}(\mathcal{N})\right)[1], L j^{*}(E)\right) \cong
\end{gathered}
$$




$$
\operatorname{Hom}_{D(X)}\left(R j_{*}\left(F \otimes S_{\mathcal{O}_{Y}}^{-l-1}(\mathcal{N}) \otimes \operatorname{det} \mathcal{N}\right)[1-c], E\right)
$$

Proof. Applying (1.8) to $i: \widetilde{Y} \rightarrow \widetilde{X}$ in place of $f$, we see that (1.17) is isomorphic to

$$
\operatorname{Hom}_{D(\widetilde{Y})}\left(L \pi^{*}(F) \otimes \mathcal{O}_{\pi}(l), L i^{*} \circ L q^{*}(E) \otimes \omega_{i}[-1]\right) .
$$

Replacing here $\omega_{i}$ by (1.12) and $L i^{*} \circ L q^{*}$ by $L \pi^{*} \circ L j^{*}$, we rewrite (1.20) as

$$
\operatorname{Hom}_{D(\widetilde{Y})}\left(L \pi^{*}(F) \otimes \mathcal{O}_{\pi}(l), L \pi^{*} \circ L j^{*}(E) \otimes \mathcal{O}_{\pi}(-1)[-1]\right) .
$$

Multiply both arguments of (1.21) by the same invertible sheaf $\pi^{*}(\operatorname{det} \mathcal{N})^{-1} \otimes$ $\mathcal{O}_{\pi}(1-c)$ and then apply to them the same shift $[c]$, without changing Hom. Using (1.15) to rewrite the new second argument, we see that the result will be

$$
\operatorname{Hom}_{D(\widetilde{Y})}\left(L \pi^{*}(F) \otimes \pi^{*}(\operatorname{det} \mathcal{N})^{-1} \otimes \mathcal{O}_{\pi}(l+1-c)[c], L \pi^{*} \circ L j^{*}(E) \otimes \omega_{\pi}[\operatorname{dim} \pi]\right)
$$

We can rewrite (1.22) using the adjunction formula (1.8) for $\pi$ :

$$
\operatorname{Hom}_{D(Y)}\left(R \pi_{*}\left[L \pi^{*}\left(F \otimes(\operatorname{det} \mathcal{N})^{-1}\right) \otimes \mathcal{O}_{\pi}(l+1-c)\right][c], L j^{*}(E)\right)
$$

Now apply the projection formula for $\pi$ (cf. [Huy], (3.11) on p. 83) to the first argument:

$$
\begin{gathered}
R \pi_{*}\left[L \pi^{*}\left(F \otimes(\operatorname{det} \mathcal{N})^{-1}\right) \otimes \mathcal{O}_{\pi}(l+1-c)\right][c] \cong \\
F \otimes(\operatorname{det} \mathcal{N})^{-1} \otimes^{L} R \pi_{*}\left(\mathcal{O}_{\pi}(l+1-c)\right)[c]
\end{gathered}
$$

and remark that the complex $R \pi_{*}\left(\mathcal{O}_{\pi}(l+1-c)\right)$ for $1-c \leq l \leq-1$ is quasiisomorphic to $R^{c-1} \pi_{*}\left(\mathcal{O}_{\pi}(l+c-1)\right)[1-c]$. Hence (1.23) will become

$$
\operatorname{Hom}_{D(Y)}\left(F \otimes(\operatorname{det} \mathcal{N})^{-1} \otimes R^{c-1} \pi_{*}\left(\mathcal{O}_{\pi}(l+1-c)\right)[1], L j^{*}(E)\right) .
$$

Finally, relative Serre's duality and (1.5) imply that in the considered range of $l$ we have

$$
R^{c-1} \pi_{*}\left(\mathcal{O}_{\pi}(l+1-c)\right) \cong S_{\mathcal{O}_{Y}}^{-l-1}(\mathcal{N}) \otimes \operatorname{det} \mathcal{N}
$$

Thus, we proved (1.18).

Multiplying both arguments by $\operatorname{det} \mathcal{N}$, shifting by $-c$ and applying (1.16), we get (1.19).

This finishes the proof. 
Remarks. (i) For $l=-1, c=2$, (1.18) becomes simply

$$
\operatorname{Hom}_{D(Y)}\left(F[1], L j^{*}(E)\right) .
$$

This is the only case that must be considered, when $c=2$. We will use this formula in sec. 3 and 4 .

(ii) We could have started a proof of Prop. 1.7 by applying formula (1.8) to $q$, rather than to $i$, thus following the diagram (1.8) clockwise rather than counterclockwise.

1.8. Reconstruction of the derived category of a blow-up. For simplicity, we continue considering the case of codimension 2 blow-up. The main result of [Or1] in this case establishes the canonical semiorthogonal composition of $D(X)$, whose components are admissible subcategories: $\left(\widetilde{D}(Y)_{-1}, D(X)_{0}\right)$, where $D(X)_{0}:=R q^{*}(D(X))$ and $\widetilde{D}(Y)_{-1}$ consists of objects $R i_{*}\left(L \pi^{*}(F) \otimes \mathcal{O}_{\pi}(-1)\right)$ where $F \in O b D(Y)$.

Together with the formula $(1.17) \cong(1.19)$ for $c=2, l=-1$, this semiorthogonal decomposition allows us to reconstruct $D(\widetilde{X})$, however, not directly, but only through the mediation of $D G$-categories, say, of the canonical enhancements of all relevant derived categories (cf. [BoKa]) and [LuOr] ).

The relevant construction on the level of DG-categories is described in [Ta], Definition 3.57, under the name "upper triangular DG-categories". Here we briefly describe its relation to the (two terms) semiorthogonal decompositions of triangular categories, as was explained to us in [Or2].

Generally, let $\mathbf{A}$ be a linear pretriangulated DG-category, and $\mathcal{A}:=H^{0}(\mathbf{A})$ its homotopy category with its natural triangulated structure. Assume that $\mathcal{A}$ admits a semiorthogonal decomposition $(\mathcal{E}, \mathcal{F})$ as above, in the case of blow-up. Lift $\mathcal{F}, \mathcal{E}$ to the subcategories $\mathbf{F}, \mathbf{E}$ of $\mathbf{A}$, and consider a pair of objects $F, E$ of the respective categories. The bifunctor given on objects by $(F, E) \mapsto \operatorname{Hom}_{\mathbf{A}}(F, E)$ with values in complexes of linear spaces can be considered as DG-functor $\Phi: \mathbf{E} \rightarrow \mathbf{M o d}-\mathbf{F}$, "the upper right corner of the upper triangular DG-category" in the sense of [Ta].

Conversely, given a triple $(\mathbf{F}, \mathbf{E}, \Phi)$ as above, one can explicitly construct a new DG-category B. Its objects are triples $(F, E, q)$ where $q \in \Phi(E)(F)^{1}$. Moreover, morphisms constitute the complex

$$
\operatorname{Hom}_{\mathbf{B}}\left((F, E, q),\left(F^{\prime}, E^{\prime}, q^{\prime}\right)\right):=\operatorname{Hom}_{\mathbf{F}}\left(F, F^{\prime}\right) \oplus \operatorname{Hom}_{\mathbf{E}}\left(E, E^{\prime}\right) \oplus \Phi\left(E^{\prime}, F\right)
$$

with pretty obvious composition rule. 
Now, the principle result is that $\mathbf{B}$ is quasiequivalent to $\mathbf{A}$.

This fact, together with uniqueness of enhancements and explicit description (1.25) of the bifunctor $\Phi$ in the blow-up case, furnishes the reconstruction of the triangulated category.

\section{Keel's tower}

2.1. Notation: combinatorics of marks. Let $S$ be a finite set, card $S=n \geq$ 3. We will call an inductive structure on $S$ the choice of a three-element subset $P \subset S$. We will sometimes denote $\Sigma:=S \backslash P$ so that $S=\Sigma \sqcup P$.

Recall that boundary strata of $\bar{M}_{0, S}$ are bijectively numbered by the (isomorphism classes of) stable $S$-marked trees. Such a tree describes the dual combinatorial type of the curve parametrized by the generic point of the respective stratum. The number of edges of such a tree equals the codimension of the stratum.

In particular, boundary divisors, that is, trees with one edge, are determined by unordered 2-partitions $S=S_{1} \sqcup S_{2}$, stable in the sense that $\left|S_{i}\right| \geq 2$. Furthermore, codimension two strata are determined by 3-partitions $S=S_{1} \sqcup S_{2} \sqcup S_{3}$ in which the middle term $S_{2}$ is uniquely defined, whereas $S_{1}$ and $S_{2}$ can be interchanged. Stability condition here means that $\left|S_{1}\right|,\left|S_{3}\right| \geq 2,\left|S_{2}\right| \geq 1$.

Whenever an inductive structure $P$ is chosen on $S$, and $|S| \geq 4$, we may and will order each stable 2-partition by the condition $\left|S_{1} \cap P\right| \leq 1$, and for $|S| \geq 4$ we will order each stable 3-partition by the condition $\left|\left(S_{1} \cup S_{2}\right) \cap P\right| \leq 1$.

2.2. Keel's blow-down. Now, assuming $n:=|S| \geq 4$ and given an inductive structure on $S$, consider a one-point set $\{\bullet\}$ disjoint from $S$ and the diagram of two forgetful morphisms, forgetting respectively sections marked by $\Sigma$ and the one marked by $\bullet$ :

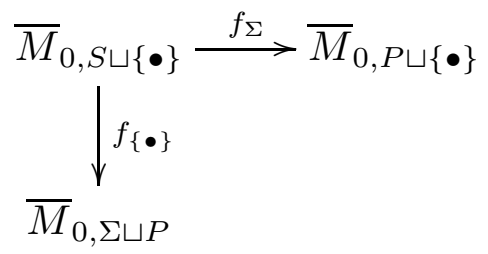

Notice that $\bar{M}_{0, P \sqcup\{\bullet\}}$ is $\mathbf{P}^{1}$ endowed with three boundary points. They are canonically marked by unordered partitions of $P \sqcup\{\bullet\}$ into two parts of cardinality 2. Such a partition, in turn, is determined by an element $p$ of $P$ (one part is $\{\bullet, p\}$ ) or a two-element subset of $P$ (the part not containing $\bullet$ ).

We summarize below some results of $[\mathrm{Ke}]$, showing, in particular, that the map (inverse to)

$$
\left(f_{\{\bullet\}}, f_{\Sigma}\right): \bar{M}_{0, S \sqcup\{\bullet\}} \rightarrow \bar{M}_{0, S} \times \bar{M}_{0, P \sqcup\{\bullet\}}
$$


is a composition of blow-ups of smooth codimension two subvarieties isomorphic to boundary divisors of $\bar{M}_{0, S}$. These blow ups naturally form a sequence of $n-3$ steps $b_{k}: B_{k, S} \rightarrow B_{k-1, S}, k=2, \ldots, n-2$. At each step, a union of pairwise disjoint connected smooth submanifolds of codimension two is blown up.

In order to bridge our notation with Keel's, the reader should have in mind the following case:

$$
S:=\{1, \ldots, n\}, \quad \bullet:=n+1, \quad P:=\{1,2,3\}, \quad \Sigma:=\{4, \ldots, n\} .
$$

2.3. Exceptional divisors of $\left(f_{\{\bullet\}}, f_{\Sigma}\right)$. In our notation, Lemma 1 of $[\mathrm{Ke}]$ establishes that exceptional divisors of the morphism $\left(f_{\{\bullet\}}, f_{\Sigma}\right)$ are exactly all boundary divisors of $\bar{M}_{0, S \sqcup\{\bullet\}}$ corresponding to the stable 2-partitions of $S \sqcup\{\bullet\}$, satisfying the following condition:

$\left(^{*}\right)$ The part containing $\bullet$ contains no more than one element of $P$ and has cardinality $\geq 3$.

As an independent check, the reader can convince oneself that the number of such partitions coincides with the difference of ranks of the Picard groups

$$
\operatorname{rkPic} \bar{M}_{0, S \sqcup\{\bullet\}}-\operatorname{rk} \operatorname{Pic}\left(\bar{M}_{0, S} \times \bar{M}_{0, P \sqcup\{\bullet\}}\right)=2^{n-1}-n-1 .
$$

Let $\bar{\sigma}$ be a partition of $S \sqcup\{\bullet\}$ satisfying (*) above, and let $\sigma$ be be the respective partition of $S$ obtained by deleting $\bullet$. Obviously, we have

$$
f_{\bullet}\left(D_{\bar{\sigma}}\right)=D_{\sigma} .
$$

We will call the cardinality of the second part of $\bar{\sigma}$ (and of $\sigma$ ) the height of $D_{\bar{\sigma}}$.

2.4. Keel's tower. The main result of $[\mathrm{Ke}]$, sec. 1, can now be stated in the following way.

The morphism (2.1) can be represented as a product of blowing-downs

$$
\bar{M}_{0, S \sqcup\{\bullet\}}=: B_{n-2, S} \rightarrow B_{n-3, S} \rightarrow \cdots \rightarrow B_{1, S}:=\bar{M}_{0, S} \times \bar{M}_{0, P \sqcup\{\bullet\}}
$$

satisfying the following conditions:

(i) Image of any exceptional divisor $D_{\bar{\sigma}}$ of height $h$ remains a divisor in $B_{h, S}$, but becomes a closed subscheme of codimension 2 in $B_{h-1, S}, \ldots, B_{1, S}$. The product of the subsequent arrows, followed by the projection $B_{1, S} \rightarrow \bar{M}_{0, S}$ identifies this subscheme with $D_{\sigma}$. 
(ii) Each morphism $B_{h+1, S} \rightarrow B_{h, S}$ is the blow up of the disjoint union of those subschemes in $B_{h, S}$ that are images of exceptional divisors of height $h+1$. Hence connected components of the center of the respective blow up are isomorphic to $\bar{M}_{0, p+1} \times \bar{M}_{0, q+1}, p, q \leq n-2$.

\section{Inductive construction of semiorthogonal decompositions and complete exceptional collections.}

3.1. The inductive step I: functoriality in $S$. In order to calculate $D^{b}\left(\bar{M}_{0, S \sqcup\{\bullet\}}\right)$ assuming that the derived categories of the respective modular spaces of smaller dimension are already known, we will apply D. Orlov's results summarized in sec. 1 to Keel's tower.

More precisely, Keel's tower depends on the choice of an inductive structure on $S$ in the sense of 2.1. In order to be able to induce a given inductive structure on the subsets of $S$, we will adopt here the following convention, essentially returning us to the Keel's choice (2.2).

$S$ is totally ordered, and for $|S| \geq 3, P \subset S$ consists of the first three elements of $S$.

The inductive structure induced on subsets of $S$ is defined then by the induced order.

With this conditions, one easily sees that a bijection of two sets of marks compatible with their respective orders lifts to a unique isomorphism of Keel's towers.

3.2. The inductive step II: Keel's blow-ups. Our "inductive leap" from $S$ to $S \sqcup\{\bullet\}$ breaks down into the sequence of small inductive steps corresponding to the consecutive floors of the Keel tower (2.4). They will allow us to obtain an inductive description of a class of semiorthogonal decompositions of $D^{b}\left(\operatorname{Coh} \bar{M}_{0, S}\right)$. Concretely, in view of Orlov's theorem, each floor $p_{k}: B_{k+1, S} \rightarrow B_{k, S}$ provides a semiorthogonal decomposition of $D^{b}\left(\operatorname{Coh} B_{k+1, S}\right)$ of the form

$$
D^{b}\left(\operatorname{Coh} B_{k+1, S}\right): \quad\left(\widetilde{D}\left(Y_{k, S}\right)_{-1}, D\left(B_{k, S}\right)_{0}\right)
$$

To be more precise, we recapitulate Orlov's results in this context. Consider the following specializations of the blow-up diagram (1.2):

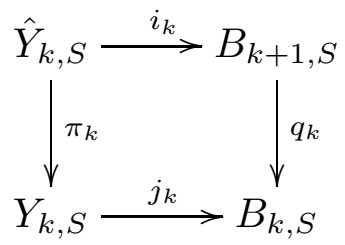


in which $i_{k}: \widehat{Y}_{k, S} \rightarrow B_{k+1, S}$ is the embedding of the exceptional divisor of $q_{k}$. Since $q_{k}$ blows up codimension two submanifolds, $\pi_{k}: \widehat{Y}_{k, S} \rightarrow Y_{k, S}$ is a fibration with fiber $\mathbf{P}^{1}$. Let $O(-1)$ be the respective relative sheaf on $\widehat{Y}_{k, S}$. Then $D^{b}\left(\operatorname{Coh} \widehat{Y}_{k, S}\right)$ has the semiorthogonal decomposition

$$
D^{b}\left(\operatorname{Coh} \widehat{Y}_{k, S}\right): \quad\left(\pi_{k}^{*}\left(D^{b}\left(\operatorname{Coh} Y_{k, S}\right)\right) \otimes O(-1), q_{k}^{*}\left(D^{b}\left(\operatorname{Coh} B_{k, S}\right)\right)\right)
$$

Now, the subcategory $\widetilde{D}\left(Y_{k, S}\right)_{-1}$ in $(3.1)$ is defined as

$$
\widetilde{D}\left(Y_{k, S}\right)_{-1}:=R i_{k *}\left[\pi_{k}^{*}\left(D^{b}\left(\operatorname{Coh} Y_{k, S}\right)\right) \otimes O(-1)\right]
$$

whereas $D\left(B_{k, S}\right)_{0}$ in $(3.1)$ is

$$
D\left(B_{k, S}\right)_{0}:=L q_{k}^{*}\left(D^{b}\left(\operatorname{Coh} B_{k, S}\right)\right) .
$$

Moreover, since $q_{k}$ blows up a disjoint union of smooth submanifolds $Y_{\sigma}, \sigma=$ $\left(S_{1}, S_{2}\right)$, each of the subcategories $\widetilde{D}\left(Y_{k, S}\right)_{-1}$ admits an orthogonal decomposition

$$
\widetilde{D}\left(Y_{k, S}\right)_{-1}: \quad\left(\widetilde{D}\left(Y_{\sigma}\right)_{-1} \mid \operatorname{card} S_{2}=k+2\right)
$$

Finally, we have a canonical identification

$$
Y_{\sigma}=\bar{M}_{0, S_{1} \sqcup\left\{\bullet_{\sigma}\right\}} \times \bar{M}_{0, S_{2} \sqcup\left\{\bullet_{\sigma}\right\}}
$$

where $\bullet_{\sigma}$ corresponds to the intersection point of two components. Therefore $D^{b}\left(\operatorname{Coh} Y_{\sigma}\right)$ is generated by an external product $\otimes$ of any two exceptional collections generating respectively $\bar{M}_{0, S_{1} \sqcup\left\{\bullet_{\sigma}\right\}}$ and $\bar{M}_{0, S_{2} \sqcup\left\{\bullet_{\sigma}\right\}}$.

This "Künneth formula" for derived and DG-categories is known on various levels of generality. Ch. Böhning ([Bö]) establishes it for collections of locally free sheaves; the proof generalizes to complexes of locally free sheaves. In [BoLaLu] the DG case is treated.

The base $B_{1, S}$ has a similar decomposition (cf. (2.4)). Thus, Keel's tower provides a tool to generate semi-orthogonal decompositions and exceptional collections for $D^{b}\left(\bar{M}_{0, n+1}\right)$ from similar objects for $D^{b}\left(\bar{M}_{0, m}\right), m \leq n$.

3.3. Exceptional collections for small $n$. On $\bar{M}_{0,4} \equiv \mathbf{P}^{1}$ the standard full strong exceptional collection is $(\mathcal{O}(-1), \mathcal{O})$.

If we represent $\bar{M}_{0,5}$ as a blow-up $p: \bar{M}_{0,5} \rightarrow \mathbf{P}^{2}$ at four points, and denote by $l_{i}, i=1, \ldots, 4$ the respective exceptional divisors, then for any choice $\left(F_{0}, F_{1}, F_{2}\right)$ 
of a full strong exceptional collection on $\mathbf{P}^{2}$, e. g. $F_{i}=\mathcal{O}(i-2)$, Orlov's theorem will provide a full strong exceptional collection on $\bar{M}_{0,5}$ of the form

$$
\left(\mathcal{O}_{l_{1}}(-1), \ldots, \mathcal{O}_{l_{4}}(-1), p^{*} F_{0}, p^{*} F_{1}, p^{*} F_{2}\right)
$$

In $[\operatorname{KarN}]$ it was shown that a sequence of mutations turns such a sequence into a full exceptional collection of locally free sheaves (this trick actually works for all del Pezzo surfaces). Taking its $\boldsymbol{\otimes}$ with the standard collection on $\mathbf{P}^{1}$ and then using Keel's blow-up, one gets an analog of (3.8) for $\bar{M}_{0,6}$.

In the remaining part of this section we will show that under certain assumptions, one can find a sequence of mutations, turning the collection thus obtained into a collection of locally free sheaves. In the next section we will demonstrate that this procedure can be applied to $\bar{M}_{0,6}$.

3.4. Preparation. Consider a blow-up diagram (1.2)

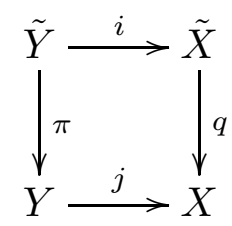

For a general blow up $R^{0} q_{*} \mathcal{O}_{\widetilde{X}} \simeq \mathcal{O}_{X}$ and $R^{i} q_{*} \mathcal{O}_{\widetilde{X}}=0$ (see [Or1], proof of Lemma 4.1). Hence the projection formula and the Leray spectral sequence give $H^{i}\left(\widetilde{X}, q^{*} \mathcal{E}\right)=H^{i}(X, \mathcal{E})$ for any locally free sheaf $\mathcal{E}$.

From now on we assume that $j(Y)$ is of codimension two in $X$. If $F_{1}, \ldots, F_{r}$ (resp. $E_{1}, \ldots, E_{n}$ ) is a full exceptional collection of locally free sheaves on $Y$ (resp. on $X)$, then Orlov's collection (1.6)

$$
R i_{*}\left(\pi^{*} F_{1} \otimes \mathcal{O}_{\pi}(-1)\right), \ldots, R i_{*}\left(\pi^{*} F_{r} \otimes \mathcal{O}_{\pi}(-1)\right), q^{*} E_{1}, \ldots, q^{*} E_{n}
$$

is a full exceptional collection on $\tilde{X}$.

3.5. Proposition. Let $E_{1}, \ldots E_{n}$ be a full exceptional collection of locally free sheaves on $X$. Assume moreover that $j^{*} E_{1}, \ldots, j^{*} E_{r}$ form a full exceptional collection on $Y$. If $Y$ is of codimension 2 in $X$, then the collection

$$
q^{*} E_{1}, q^{*} E_{1}(\tilde{Y}), \ldots, q^{*} E_{r}, q^{*} E_{r}(\widetilde{Y}), q^{*} E_{r+1}, \ldots, q^{*} E_{n}
$$

is a full exceptional collection on $\widetilde{X}$. 
Here and below we use notation of the type $q^{*} L(\tilde{Y})$ as a shorthand for $q^{*} L \otimes$ $\mathcal{O}_{\widetilde{X}}(i(\widetilde{Y}))$.

Proof. The strategy of our proof is simple. We start with the exceptional collection (3.9), with $F_{a}:=q^{*} E_{a}$ for $1 \leq a \leq r$, and show that it can be transformed into (3.10) by an explicit sequence of mutations (cf. [Bo], [Kuz]).

More precisely, for $1 \leq a \leq r$, put $A_{a}:=R i_{*}\left(\pi^{*} j^{*} E_{a} \otimes \mathcal{O}_{\pi}(-1)\right), B_{a}:=q^{*} E_{a}$.

We will first check that

$$
\operatorname{Hom}^{\bullet}\left(A_{b}, B_{a}\right)=0 \quad \text { for } \quad b>a .
$$

so that the right mutation of such an exceptional pair simply reduces to the permutation $\left(A_{b}, B_{a}\right) \mapsto\left(B_{a}, A_{b}\right)$. This shows, that we may consecutively move $A_{r}$ in (3.9) to the right, until it reaches the position directly to the left of $B_{r}$; then move $A_{r-1}$ to the right, until it reaches the position directly to the left of $B_{r-1}$; and so on. The result will be the exceptional collection $\left(A_{1}, B_{1} ; A_{2}, B_{2} ; \ldots ; A_{r}, B_{r} ; B_{r+1}, \ldots, B_{n}\right)$.

Second, we will check that

$$
R_{B_{a}}\left(A_{a}\right) \cong B_{a}(\tilde{Y})
$$

Thus additional $r$ right mutations will transform the latter collection into

$$
\left.\left(B_{1}, B_{1}(\widetilde{Y})\right), \ldots, B_{r}, B_{r}(\widetilde{Y}), B_{r+1}, \ldots, B_{n}\right)
$$

that is, to $(3.10)$.

Proof of (3.11). Consider the isomorphism (1.17) $\cong(1.25)$ written for $F:=$ $j^{*} E_{b}, E:=E_{a}[i]$ where $i$ is an arbitrary shift and $b>a$. Its left hand side will represent one of the components of $\operatorname{Hom}^{\bullet}\left(A_{b}, B_{a}\right)$. Hence it suffices to prove that the right hand side vanishes. But it is simply $\operatorname{Hom}_{D(Y)}\left(j^{*}\left(E_{b}\right)[1], j^{*} E_{a}[i]\right)$, $1 \leq a \leq b \leq r$, and all these groups vanish, because we assumed that $j^{*} E_{1}, \ldots, j^{*} E_{r}$ is an exceptional collection.

Proof of (3.12). Consider now the case $a=b$. First of all, recall that $R_{B_{a}}\left(A_{a}\right)$ is defined as the cone $C\left(\alpha_{a}\right)$ of the canonical morphism in $D(\widetilde{X})$

$$
\alpha_{a}: A_{a} \rightarrow \operatorname{Hom}^{\bullet}\left(A_{a}, B_{a}\right)^{t} \otimes B_{a}
$$

where $t$ means linear dual in the category of of graded linear spaces. In order to calculate $\operatorname{Hom}^{\bullet}\left(A_{a}, B_{a}\right)$, we note that $A_{a}$ fits into exact sequence $0 \rightarrow q^{*} E_{a} \rightarrow$ 
$q^{*} E_{a}(\tilde{Y}) \rightarrow A_{a} \rightarrow 0$ (cf. (1.13)), and thus $A_{a}$ is quasi-isomorphic to its projective resolution

$$
\mathcal{A}_{a}: 0 \rightarrow q^{*} E_{a} \rightarrow q^{*} E_{a}(\widetilde{Y}) \rightarrow 0
$$

(with the first term in degree -1 ).

Since $A_{a}$ is exceptional, $\operatorname{Hom}^{\bullet}\left(\mathcal{A}_{a}, B_{a}\right)$ is spanned by the canonical isomorphism $\mathcal{A}_{a} \rightarrow B_{a}[1]$ which is identity in degree -1 , and the morphism (3.13) can be represented by the morphism of complexes

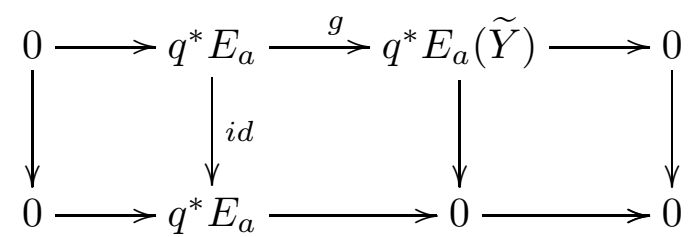

The cone of it is the complex

$$
0 \longrightarrow q^{*} E_{a} \stackrel{(i d,-g)}{\longrightarrow} q^{*} E_{a} \oplus q^{*} E_{a}(\tilde{Y}) \longrightarrow 0
$$

where $q^{*} E_{a} \oplus q^{*} E_{a}(\widetilde{Y})$ is in degree -1 . There exists a short exact sequence of sheaves

$$
0 \longrightarrow q^{*} E_{a} \stackrel{(i d,-g)}{\longrightarrow} q^{*} E_{a} \oplus q^{*} E_{a}(\tilde{Y}) \stackrel{\psi}{\longrightarrow} q^{*} E_{a}(\tilde{Y}) \longrightarrow 0
$$

where $\psi(v, w)=g(v)+w$. Therefore, the cone is quasi-isomorphic to the complex with one non-zero term $q^{*} E(\widetilde{Y})$ placed in degree -1 , i.e. $q^{*} E_{a}(\widetilde{Y})[1]$. Hence $R_{B_{a}}\left(A_{a}\right)[1] \cong q^{*} E_{a}(\tilde{Y})[1]$ and finally $R_{B_{a}}\left(A_{a}\right) \cong B_{a}(\tilde{Y})$.

This completes the proof of Proposition 3.5.

3.6. Corollary. Let $E_{1}, \ldots, E_{n}$ be a full exceptional collection of locally free sheaves on $X$. Assume moreover that for some $s \leq r, j^{*} E_{s}, \ldots, j^{*} E_{r}$ form a full exceptional collection on $Y$. If $Y$ is of codimension 2 in $X$, then the collection

$$
q^{*} E_{1}(\tilde{Y}), \ldots, q^{*} E_{s-1}(\tilde{Y}), q^{*} E_{s}, q^{*} E_{s}(\tilde{Y}), \ldots, q^{*} E_{r}, q^{*} E_{r}(\tilde{Y}), q^{*} E_{r+1}, \ldots, q^{*} E_{n}
$$

is a full exceptional collection on $\widetilde{X}$.

Proof. First, let us recall a general fact. Let $\mathcal{D}$ be a triangulated category with a Serre functor $S: \mathcal{D} \rightarrow \mathcal{D}$ and $\mathcal{A}$ an admissible subcategory (cf. [BoKa1], [Kuz]). In 
this situation we have two semi-orthogonal decompositions $\left\langle\mathcal{A},{ }^{\perp} \mathcal{A}\right\rangle$ and $\left\langle\mathcal{A}^{\perp}, \mathcal{A}\right\rangle$. By [BoKa1], Proposition 3.6, we obtain that

$$
\begin{gathered}
R_{\perp \mathcal{A}}(\mathcal{A})=S^{-1}(\mathcal{A}), \\
L_{\mathcal{A}^{\perp}}(\mathcal{A})=S(\mathcal{A}) .
\end{gathered}
$$

Our proof of corollary 3.6 will consist of applications of formulas (3.13),(3.14) and proposition 3.5.

Let $\mathcal{D}=D(X), \mathcal{A}=\left\langle E_{1}, \ldots, E_{s-1}\right\rangle,{ }^{\perp} \mathcal{A}=\left\langle E_{s}, \ldots, E_{n}\right\rangle$ and the Serre functor is $S_{X}=\cdot \otimes \omega_{X}[\operatorname{dim} X]$. Applying formula $(3.13)$ we get a full exceptional collection

$$
E_{s}, \ldots, E_{n}, S_{X}^{-1}\left(E_{1}\right), \ldots, S_{X}^{-1}\left(E_{s-1}\right)
$$

To this collection we can apply proposition 3.5 and we get a full exceptional collection on $\widetilde{X}$

$q^{*} E_{s}, q^{*} E_{s}(\widetilde{Y}), \ldots, q^{*} E_{r}, q^{*} E_{r}(\tilde{Y}), q^{*} E_{r+1}, \ldots, q^{*} E_{n}, L q^{*} \circ S_{X}^{-1}\left(E_{1}\right), \ldots, L q^{*} \circ S_{X}^{-1}\left(E_{s-1}\right)$

Let now $\mathcal{D}=D(\tilde{X}), \mathcal{A}=\left\langle L q^{*} S_{X}^{-1}\left(E_{1}\right), \ldots, L q^{*} S_{X}^{-1}\left(E_{s-1}\right)\right\rangle$ and the Serre functor is $S_{\widetilde{X}}=\cdot \otimes \omega_{\widetilde{X}}[\operatorname{dim} \widetilde{X}]$. Applying formula (3.14) and using that

$$
S_{\widetilde{X}} \circ L q^{*} \circ S_{X}^{-1} \simeq L q^{*} \otimes \mathcal{O}_{\widetilde{X}}(\widetilde{Y})
$$

we get the desired statement. 


\section{Example: moduli space $\bar{M}_{0,6}$}

4.1. Preparation: moduli space $\bar{M}_{0,5}$. Let $S=\{1,2,3,4,5\}, S^{\prime}=\{1,2,3,4\}$ and $P=\{1,2,3\}$. Consider the Keel's tower

$$
\begin{gathered}
B_{2, S^{\prime}}=\bar{M}_{0, S} \\
\downarrow^{q_{1, S^{\prime}}} \\
B_{1, S^{\prime}}=\bar{M}_{0, S^{\prime}} \times \bar{M}_{0, P \sqcup\{5\}}
\end{gathered}
$$

The map $q_{1, S^{\prime}}$ contracts 3 boundary divisors $D_{\sigma_{i}}$ corresponding to unordered partitions

$$
\sigma_{1}=(5,1,4 \mid 2,3), \quad \sigma_{2}=(5,2,4 \mid 1,3), \quad \sigma_{3}=(5,3,4 \mid 2,1) .
$$

Let us identify $\bar{M}_{0, S^{\prime}}$ with $\bar{M}_{0, P \sqcup\{5\}}$ using the bijection of labels identical on $\{1,2,3\}$ and mapping 4 to 5 . Then images of $D_{\sigma_{i}}$ become three points on the diagonal, corresponding to the partitions with one part $\{i, 4\}$, resp. $\{i, 5\}$. Let us imagine $\bar{M}_{0, P \sqcup\{5\}}$ as the horizontal axis $\mathbf{P}^{1}$, and $\bar{M}_{0, S^{\prime}}$ as the vertical one. Let $H_{i}$, resp. $V_{i}$, be the horizontal, resp. vertical fiber, passing through the image of $D_{\sigma_{i}}$.

Now denote by $\widetilde{H}_{i}$ and $\widetilde{V}_{i}$ the proper transforms of $H_{i}$, resp. $V_{i}$, in $\bar{M}_{0, S}$, and let $\widetilde{Z}$ be the proper transform of the diagonal $Z$. Divisors $D_{\sigma_{i}}, \widetilde{H}_{i}, \widetilde{V}_{i}$ and $\widetilde{Z}$ are all isomorphic to $\mathbf{P}^{1}$ and have self-intersection $(-1)$. There are precisely10 such curves on $\bar{M}_{0, S}$.

Let $F_{0}, F_{1}$ and $G_{0}, G_{1}$ be full exceptional collections of locally free sheaves on $\bar{M}_{0, S^{\prime}}$ and $\bar{M}_{0, P \sqcup\{5\}}$ respectively. It is well known that $F_{i}, G_{i}$ invertible sheaves. We know that

$$
F_{0} \otimes G_{0}, F_{1} \otimes G_{0}, F_{0} \otimes G_{1}, F_{1} \otimes G_{1}
$$

is a full exceptional collection on $\bar{M}_{0, S^{\prime}} \times \bar{M}_{0, P \sqcup\{5\}}$. Denote it as

$$
L_{0}, L_{1}, L_{2}, L_{3} \text {. }
$$

Consider the decomposition $q_{1, S^{\prime}}=f_{1} \circ f_{2} \circ f_{3}$ where $f_{i}$ contracts only $D_{\sigma_{i}}$. Each $f_{i}$ is a blow-up of a surface at a point. Applying corollary 3.6 to the blow-up $f_{3}$ and using restriction of $L_{0}$ as a full exceptional collection in $D(p t)$ we obtain a full exceptional collection on the resulting surface

$$
f_{3}^{*} L_{0}, f_{3}^{*} L_{0}\left(D_{\sigma_{3}}\right), f_{3}^{*} L_{1}, f_{3}^{*} L_{2}, f_{3}^{*} L_{3}
$$


Continuing in the same way and always using restriction of the first element as a full exceptional collection in $D(p t)$ we obtain a full exceptional collection on $\bar{M}_{0, S}$

$$
q_{1, S^{\prime}}^{*} L_{0}, q_{1, S^{\prime}}^{*} L_{0}\left(D_{\sigma_{1}}\right), q_{1, S^{\prime}}^{*} L_{0}\left(D_{\sigma_{2}}\right), q_{1, S^{\prime}}^{*} L_{0}\left(D_{\sigma_{3}}\right), q_{1, S^{\prime}}^{*} L_{1}, q_{1, S^{\prime}}^{*} L_{2}, q_{1, S^{\prime}}^{*} L_{3} .
$$

Of course, this is just an example of a full exceptional collection on $\bar{M}_{0, S}$. If we used restrictions of other elements of (4.1) we would have obtained a different answer.

4.2. Preparation: moduli space $\bar{M}_{0,6}$. Here we will introduce convenient notations which will be used later to write down a full exceptional collection on $\bar{M}_{0,6}$.

Let $S=\{1,2,3,4,5\},\{\bullet\}=\{6\}$ and $P=\{1,2,3\}$. Consider the Keel's tower

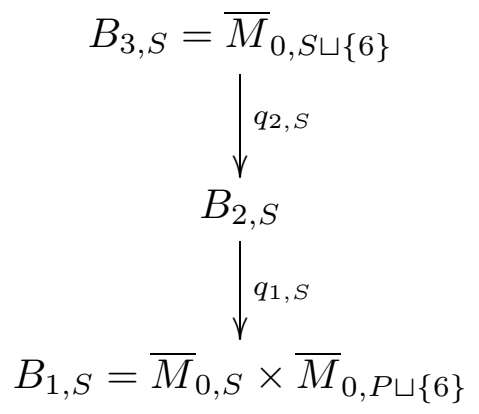

The map $q_{1, S} \circ q_{2, S}$ contracts 10 boundary divisors $E_{i}, 1 \leq i \leq 10$. At the height 3 level it contracts 7 boundary divisors corresponding to the following unordered partitions

$$
\begin{aligned}
& E_{4} \leftrightarrow(6,1,4 \mid 5,2,3), \quad E_{6} \leftrightarrow(6,2,4 \mid 5,1,3), \quad E_{8} \leftrightarrow(6,3,4 \mid 5,2,1), \\
& E_{5} \leftrightarrow(6,1,5 \mid 4,2,3), \quad E_{7} \leftrightarrow(6,2,5 \mid 4,1,3), \quad E_{9} \leftrightarrow(6,3,5 \mid 4,2,1),
\end{aligned}
$$

and $E_{10} \leftrightarrow(6,4,5 \mid 1,2,3)$. At the height 2 level it contracts images under $q_{2, S}$ of 3 boundary divisors corresponding to the following unordered partitions

$$
E_{1} \leftrightarrow(6,1,4,5 \mid 2,3), \quad E_{2} \leftrightarrow(6,2,4,5 \mid 1,3), \quad E_{3} \leftrightarrow(6,3,4,5 \mid 2,1)
$$

The divisors $E_{1}, E_{2}, E_{3}$ are pairwise disjoint and $E_{4}, \ldots E_{10}$ are pairwise disjoint as well. We list below all nonempty intersections

$$
E_{1} \cdot E_{4}=P_{1}, \quad E_{1} \cdot E_{5}=Q_{1}
$$




$$
\begin{gathered}
E_{2} \cdot E_{6}=P_{2}, \quad E_{2} \cdot E_{7}=Q_{2}, \\
E_{3} \cdot E_{8}=P_{3}, \quad E_{3} \cdot E_{9}=Q_{3}, \\
E_{1} \cdot E_{10}=R_{1} ; \quad E_{2} \cdot E_{10}=R_{2} ; \quad E_{3} \cdot E_{10}=R_{3},
\end{gathered}
$$

where $P_{i}, Q_{i}, R_{i}$ are isomorphic to $\mathbf{P}^{1}$ and all intersections are transversal.

Let $L_{0}, \ldots, L_{6}$ be an exceptional collection of invertible sheaves on $\bar{M}_{0, S}$ and $G_{0}, G_{1}$ an exceptional collection of invertible sheaves on $\bar{M}_{0, P \sqcup\{6\}}$. From them we construct a collection on $\bar{M}_{0, S} \times \bar{M}_{0, P \sqcup\{6\}}$

$$
L_{0} \otimes G_{0}, \ldots L_{6} \otimes G_{0}, L_{0} \otimes G_{1}, \ldots L_{6} \otimes G_{1} .
$$

We will need some assumptions on the collection $L_{0}, \ldots, L_{6}$. We use notations for $\bar{M}_{0, S^{\prime}}$ introduced earlier in section 4.1.

Assumption 1. $L_{1}, L_{2}$ restricted to $D_{\sigma_{1}}$ form a full exceptional collection on $D_{\sigma_{1}}$; $L_{2}, L_{3}$ restricted to $D_{\sigma_{2}}$ form a full exceptional collection $D_{\sigma_{2}} ; L_{3}, L_{4}$ restricted to $D_{\sigma_{3}}$ form a full exceptional collection $D_{\sigma_{3}}$.

Assumption 2. $L_{0}, L_{1}$ restrict to a full exceptional collection on $\widetilde{H}_{1}$ and $\widetilde{V}_{1}$. The same holds for $L_{1}, L_{2}$ on $\widetilde{H}_{2}, \widetilde{V}_{2}$ and $L_{2}, L_{3}$ on $\widetilde{H}_{3}, \widetilde{V}_{3}$ and $L_{5}, L_{6}$ on $\widetilde{Z}$.

These assumptions are satisfied, for example, for

$$
\mathcal{O}, \mathcal{O}\left(D_{\sigma_{1}}\right), \mathcal{O}\left(D_{\sigma_{2}}\right), \mathcal{O}\left(D_{\sigma_{3}}\right), q_{1, S^{\prime}}^{*} \mathcal{O}(0,1), q_{1, S^{\prime}}^{*} \mathcal{O}(1,0), q_{1, S^{\prime}}^{*} \mathcal{O}(1,1)
$$

where we used notations of section 4.1 and identification of $\bar{M}_{0, S^{\prime}} \times \bar{M}_{0, P \sqcup\{5\}}$ with $\mathbf{P}^{1} \times \mathbf{P}^{1}$.

4.3. Collection. Similar to section 4.1, in view of Keel's tower (4.3), consecutively applying corollary 3.6 to full exceptional collection (4.4) one can obtain a full exceptional collection on $\bar{M}_{0,6}$. We start with listing its elements, and afterwards give some indications about how it was obtained.

Let $q=q_{1, S} \circ q_{2, S}$ and $E_{\geq i}=\sum_{k=i}^{k=10} E_{k}$. The exceptional collection is

$$
\begin{gathered}
q^{*}\left(L_{0} \otimes G_{0}\right)\left(E_{\geq 1}\right), \\
q^{*}\left(L_{1} \otimes G_{0}\right)\left(E_{\geq 2}\right), q^{*}\left(L_{1} \otimes G_{0}\right)\left(E_{\geq 1}\right), \\
q^{*}\left(L_{2} \otimes G_{0}\right)\left(E_{\geq 2}\right), q^{*}\left(L_{2} \otimes G_{0}\right)\left(E_{1}+E_{\geq 3}\right), q^{*}\left(L_{2} \otimes G_{0}\right)\left(E_{\geq 1}\right),
\end{gathered}
$$




$$
\begin{gathered}
q^{*}\left(L_{3} \otimes G_{0}\right)\left(E_{\geq 3}\right), q^{*}\left(L_{3} \otimes G_{0}\right)\left(E_{2}+E_{\geq 4}\right), q^{*}\left(L_{3} \otimes G_{0}\right)\left(E_{\geq 2}\right), \\
q^{*}\left(L_{4} \otimes G_{0}\right)\left(E_{\geq 4}\right), q^{*}\left(L_{4} \otimes G_{0}\right)\left(E_{\geq 3}\right), \\
q^{*}\left(L_{5} \otimes G_{0}\right)\left(E_{\geq 4}\right), \\
q^{*}\left(L_{6} \otimes G_{0}\right)\left(E_{\geq 4}\right), \\
q^{*}\left(L_{0} \otimes G_{1}\right)\left(E_{\geq 5}\right), q^{*}\left(L_{0} \otimes G_{1}\right)\left(E_{4}+E_{\geq 6}\right), q^{*}\left(L_{0} \otimes G_{1}\right)\left(E_{\geq 4}\right), \\
q^{*}\left(L_{1} \otimes G_{1}\right)\left(E_{\geq 6}\right), q^{*}\left(L_{1} \otimes G_{1}\right)\left(E_{\geq 5}\right), q^{*}\left(L_{1} \otimes G_{1}\right)\left(E_{4}+E_{\geq 7}\right), q^{*}\left(L_{1} \otimes G_{1}\right)\left(E_{4}+E_{6}+E_{\geq 8}\right), \\
q^{*}\left(L_{2} \otimes G_{1}\right)\left(E_{\geq 8}\right), q^{*}\left(L_{2} \otimes G_{1}\right)\left(E_{\geq 7}\right), q^{*}\left(L_{2} \otimes G_{1}\right)\left(E_{6}+E_{\geq 9}\right), q^{*}\left(L_{2} \otimes G_{1}\right)\left(E_{6}+E_{8}+E_{10}\right), \\
q^{*}\left(L_{3} \otimes G_{1}\right)\left(E_{6}+E_{\geq 8}\right), \\
\left.q_{10}\right), q^{*}\left(L_{3} \otimes G_{1}\right)\left(E_{\geq 9}\right), q^{*}\left(L_{3} \otimes G_{1}\right)\left(E_{8}+E_{10}\right), \\
q^{*}\left(L_{4} \otimes G_{1}\right)\left(E_{10}\right), \\
q^{*}\left(L_{6} \otimes G_{1}\right), q^{*}\left(L_{5} \otimes G_{1}\right), q^{*}\left(L_{6} \otimes G_{1}\right)\left(E_{10}\right),
\end{gathered}
$$

Below we describe the algorithm that was used to obtain this collection.

4.3.1 Algorithm, step I. Due to Assumption 1, the restriction of the pair

$$
L_{1} \otimes G_{0}, L_{2} \otimes G_{0}
$$

to $q\left(E_{1}\right)$ is a full exceptional collection. The same holds for pairs $L_{2} \otimes G_{0}, L_{3} \otimes G_{0}$ on $q\left(E_{2}\right)$ and $L_{3} \otimes G_{0}, L_{4} \otimes G_{0}$ on $q\left(E_{3}\right)$.

Represent $q_{1, S}=f_{1} \circ f_{2} \circ f_{3}$ as a composition of three blow-ups (cf. section 4.1) in such a way that the (preimage of) $q\left(E_{i}\right)$ is blown up at the $i$-th step for $1 \leq i \leq 3$. At the first step we use the pair $L_{1} \otimes G_{0}, L_{2} \otimes G_{0}$ to apply Corollary 3.6. At the second step we use the pair

$$
f_{1}^{*}\left(L_{2} \otimes G_{0}\right)\left(f_{2} \circ f_{3} \circ q_{2, S}\left(E_{1}\right)\right), f_{1}^{*}\left(L_{3} \otimes G_{0}\right),
$$

which restricts to a full exceptional collection on $f_{2} \circ f_{3} \circ q_{2, S}\left(E_{2}\right)$ because $q\left(E_{1}\right)$ and $q\left(E_{2}\right)$ are disjoint and $L_{2} \otimes G_{0}, L_{3} \otimes G_{0}$ restricts to an exceptional collection on $q\left(E_{2}\right)$ as was pointed out above. 
One proceeds similarly at the third step and obtains the following exceptional collection on $B_{2, S}$

$$
\begin{gathered}
q_{1, S}^{*}\left(L_{0} \otimes G_{0}\right)\left(E_{1}^{\prime}+E_{2}^{\prime}+E_{3}^{\prime}\right), \\
q_{1, S}^{*}\left(L_{1} \otimes G_{0}\right)\left(E_{2}^{\prime}+E_{3}^{\prime}\right), q_{1, S}^{*}\left(L_{1} \otimes G_{0}\right)\left(E_{1}^{\prime}+E_{2}^{\prime}+E_{3}^{\prime}\right), \\
q_{1, S}^{*}\left(L_{2} \otimes G_{0}\right)\left(E_{2}^{\prime}+E_{3}^{\prime}\right), q_{1, S}^{*}\left(L_{2} \otimes G_{0}\right)\left(E_{1}^{\prime}+E_{3}^{\prime}\right), q_{1, S}^{*}\left(L_{2} \otimes G_{0}\right)\left(E_{1}^{\prime}+E_{2}^{\prime}+E_{3}^{\prime}\right), \\
q_{1, S}^{*}\left(L_{3} \otimes G_{0}\right)\left(E_{3}^{\prime}\right), q_{1, S}^{*}\left(L_{3} \otimes G_{0}\right)\left(E_{2}^{\prime}\right), q_{1, S}^{*}\left(L_{3} \otimes G_{0}\right)\left(E_{2}^{\prime}+E_{3}^{\prime}\right), \\
q_{1, S}^{*}\left(L_{4} \otimes G_{0}\right), q_{1, S}^{*}\left(L_{4} \otimes G_{0}\right)\left(E_{3}^{\prime}\right), \\
q_{1, S}^{*}\left(L_{5} \otimes G_{0}\right), q_{1, S}^{*}\left(L_{6} \otimes G_{0}\right), \\
q_{1, S}^{*}\left(L_{0} \otimes G_{1}\right), q_{1, S}^{*}\left(L_{1} \otimes G_{1}\right), \ldots,
\end{gathered}
$$

where $E_{i}^{\prime}=q_{2, S}\left(E_{i}\right)$.

4.3.2 Algorithm, step II. Due to Assumption 2, the restriction of the pair

$$
L_{0} \otimes G_{1}, L_{1} \otimes G_{1}
$$

to $q_{2, S}\left(E_{4}\right)$ and $q_{2, S}\left(E_{5}\right)$ gives full exceptional collections on them. The same holds for the pair $L_{1} \otimes G_{1}, L_{2} \otimes G_{1}$ on $q_{2, S}\left(E_{6}\right)$ and $q_{2, S}\left(E_{7}\right)$; for $L_{2} \otimes G_{1}, L_{3} \otimes G_{1}$ on $q_{2, S}\left(E_{8}\right)$ and $q_{2, S}\left(E_{9}\right)$; for $L_{5} \otimes G_{1}, L_{6} \otimes G_{1}$ on $q_{2, S}\left(E_{10}\right)$.

Represent $q_{2, S}=g_{4} \circ \cdots \circ g_{10}$ as the composition of blow-downs $g_{i}$ of $E_{i}$ for $4 \leq i \leq 10$. To apply Corollary 3.6 to a single blow-up one needs to choose an exceptional pair. We always choose pairs related to those described in the beginning of this section(in fact, they are pull-backs of those followed by a twist with $\mathcal{O}(D)$, where $D$ is a divisor disjoint from the exceptional divisor considered at this step).

\section{References}

[AuKaOr] D. Auroux, L. Katzarkov, D. Orlov. Mirror symmetry for del Pezzo surfaces: vanishing cycles and coherent sheaves. Invent. Math. 166(2006), no. 3, 537-582. arXiv:math/0506166

[Bo] A. Bondal. Representations of associative algebras and coherent sheaves. Math. USSR Izv. 34 (1990), 23-42.

[BoKa1] A. Bondal, M. Kapranov. Representable functors, Serre functors, and reconstructions. (Russian) Izv. Akad. Nauk SSSR Ser. Mat. 53 (1989), no. 6, 1183-1205; translation in Math. USSR Izv. 35 (1990), no. 3, 519-541 
[BoKa2] A. Bondal, M. Kapranov. Framed triangulated categories. Math USSR Sbornik, 70, Nr 1 (1991), 93-107.

[BoLaLu] A. Bondal, M. Larsen, V. Lunts. Grothendieck ring of pretriangulated categories. Int. Math. Res. Notes, 29 (2004), 1461-1495.

[Bö] Ch. Böhning. Derived categories of coherent sheaves on rational homogeneous manifolds. Documenta Mathematica, 11 (2006), 261-331.

[Huy] D. Huybrechts. Fourier-Mukai transforms in algebraic geometry. Oxford UP, 2006.

[Ka] M. Kapranov. Veronese curves and Grothendieck-Knudsen moduli space $\bar{M}_{0, n}$. J. Algebraic Geom. 2 (1993), No. 2, 239-262.

[KarN] B. V. Karpov, D. Yu. Nogin. Three-block exceptional collections over del Pezzo surfaces. Izv. Math. 62 (1998), no. 3, 429-463. arXiv:alg-geom/9703027

[Ke] S. Keel. Intersection theory of moduli space of stable $N$-pointed curves of genus zero. Trans. AMS, Vol. 330, No. 2 (1992), 545-574.

[KeTe] S. Keel, J. Tevelev. Equations for $\bar{M}_{0, n}$. Int. Journ. Math., Vol. 20, No. 9 (2009), 1159-1184.

[Kel] B. Keller. On differential graded categories. International Congress of Mathematicians. Vol. II, 151-190, Eur. Math. Soc., Zrich, 2006. arXiv:0601185

[Kuz] A. Kuznetsov. Derived categories of cubic fourfolds. In: Cohomological and geometric approaches to rationality problems, Progr. Math., 282, Birkhuser Boston, Inc., Boston, MA, 2010, 219-243, arXiv:0808.3351

[LuOr] V. Lunts, D. Orlov. Uniqueness of enhancement for triangulated categories. Journ. of the AMS, vol. 23, No 3 (2010), 853-908.

[MaS] Yu. Manin, M. Smirnov. Towards motivic quantum cohomology of $\bar{M}_{0, S}$. arXiv:1107.4915

[Or1] D. Orlov. Projective bundles, monoidal transformations, and derived categories of coherent sheaves. Russian Acad. Sci. Izv. Math., vol. 41 (1993), No. 1, $133-141$.

[Or2] D. Orlov. Letter to Yu. Manin of 19/09/2011.

[Ta] G. Tabuada. Théorie homotopique des DG-catégories. arXiv:0710.4303 